\section{D) Check for updates}

Cite this: Nanoscale, 2021, 13, 8639

\title{
Ligand-dependent nano-mechanical properties of CdSe nanoplatelets: calibrating nanobalances for ligand affinity monitoring $\dagger$
}

\author{
Quentin Martinet, ${ }^{a}$ Justine Baronnier, ${ }^{a}$ Adrien Girard ${ }^{\mathrm{b}}$ Tristan Albaret, ${ }^{a}$ \\ Lucien Saviot, (D) ${ }^{c}$ Alain Mermet, ${ }^{a}$ Benjamin Abecassis, (D) d Jérémie Margueritat (D) *a \\ and Benoît Mahler*a
}

\begin{abstract}
The influence of ligands on the low frequency vibration of cadmium selenide colloidal nanoplatelets of different thicknesses is investigated using resonant low frequency Raman scattering. The strong vibration frequency shifts induced by ligand modifications as well as sharp spectral linewidths make low frequency Raman scattering a tool of choice to follow ligand exchange as well as the nano-mechanical properties of the NPLs, as evidenced by a carboxylate to thiolate exchange study. Apart from their molecular weight, the nature of the ligands, such as the sulfur to metal bond of thiols, induces a modification of the NPLs as a whole, increasing the thickness by one monolayer. Moreover, as the weight of the ligands increases, the discrepancy between the mass-load model and the experimental measurements increase. These effects are all the more important when the number of layers is small and can only be explained by a modification of the longitudinal sound velocity. This modification takes its origin in a change of the lattice structure of the NPLs, that reflects on their elastic properties. These nanobalances are finally used to characterize ligand affinity with the surface using binary thiol mixtures, illustrating the potential of low frequency Raman scattering to finely characterize nanocrystal surfaces.
\end{abstract}

Received 14th January 2021 Accepted 15th April 2021

DOI: $10.1039 / \mathrm{d} 1 \mathrm{nr} 00270 \mathrm{~h}$

rsc.li/nanoscale coupling. ${ }^{4,11}$ Phonons usually scrutinized in these cases are optical phonons, at frequencies higher than $100 \mathrm{~cm}^{-1}$.

Lower frequency vibrations also exist and are characteristic of deformations of the nanocrystal as a whole. They are related to propagative acoustic phonons in the bulk material whose velocity, the sound velocity, is directly related to the physical properties of the material, namely its mass density and elastic constants. For small enough sizes, the vibrations are confined inside the nanocrystals and some of them can inelastically scatter light and manifest as peaks in the low-frequency Raman spectra. They are known as Lamb modes. ${ }^{12}$ Their frequencies depend on the physical properties of the material as in the case of the acoustic waves, but also on the shape, size and environment of the nanocrystal. They can therefore provide crucial information about the shape of heterostructures in a non-destructive manner, as demonstrated in the case of dot-in-rod CdSe/CdS nanocrystals. ${ }^{10}$

Recent reports about low frequency phonons in nanocrystals ${ }^{13-17}$ measured using diverse techniques (pumpprobe and Raman) have also shown the importance of the surface energy of the nano-object. Indeed, controlling the surface states is crucial to control electron-phonon interactions occurring at nanocrystal surfaces. However, if the effect of the ligands on the electronic properties has been 
widely investigated, the mechanisms involved in the modifications of the phonon's frequencies are still unclear, especially in the case of the Lamb modes. This is due to two main reasons. First, the size of the nano-object must be small to observe a measurable effect of the ligands. ${ }^{13,15,18}$ Second, the study of small nanoparticles requires to study the assemblies of nanoparticles presenting a small size and shape dispersion. Indeed, the width of the measured low frequency Raman peak is rapidly enlarged with these morphological dispersion parameters ${ }^{19}$ and the effect of the ligands might be obscured. We have shown that nanoplatelets (NPLs) of CdS and CdSe are ideal objects to investigate this effect. ${ }^{15,16}$ Their thicknesses are perfectly controlled at the atomic scale from 3 to 5 atomic layers ( $0.9 \mathrm{~nm}$ to $1.5 \mathrm{~nm}$ in the case of CdSe), and due to their lateral dimension which is much larger than their thickness, only the breathing vibration of the thickness is measured, making them model nano-objects with negligible size dispersion. Moreover, in these 2D systems, the ligand nature can be easily modified through standard ligand exchange procedures. $^{20-22}$

We demonstrated previously ${ }^{15}$ that in the framework of continuum elasticity, the frequency of the breathing vibration of a free CdSe NPL depends on its density $\rho$ and elastic constant $C$ ( $C_{11}$ in the case of the cubic structure) according to

$$
\nu=\frac{1}{2 h} \sqrt{\frac{C}{\rho}}
$$

where $h=\left(a_{0} \times\right.$ number of atomic layers $)$ is the thickness of the NPL expressed as a function of the lattice parameter $a_{0}$ and $\sqrt{\frac{C}{\rho}}=v_{\mathrm{L}}$, i.e. the longitudinal speed of sound in the NPL. The presence of the native ligands can be taken into account through the inertial mass load they induce. ${ }^{15,16}$ The lumped mass effect is accounted for with the following equation, whose roots give the resonance frequencies of the NPL across the thickness:

$$
\cos \left(\frac{\omega}{V_{\mathrm{L}}} \frac{h}{2}\right)=\frac{\sigma \omega}{\rho V_{\mathrm{L}}} \sin \left(\frac{\omega}{V_{\mathrm{L}}} \frac{h}{2}\right)
$$

where $\sigma$ is the surface mass density of the ligands and $\rho$ is the volume mass density of the NPL. The magnitude of this effect is driven by the ratio of the masses between the ligands and the NPL ( $\sigma / \rho$ factor).

In this article, we demonstrate that other parameters play a role in the change of frequency and that an improved model is required. We show that the breathing vibration of NPLs also depends on the structural modification of the NPLs induced by the presence of ligands at their surfaces. This provides a new mean to control phonons in such nanostructures but also a new tool to characterize ligand induced modification of their lattice structure. This latter effect is all the more important when the NPLs are thin, i.e. when the proportion of atoms close to the surface is large. Significant deviations from the mass-load model are reported. They are explained by the modification of the NPL structure as a function of ligand nature, which impacts on the mechanical properties of the NPLs. The fine characterization of this modification allows the calibration of the NPL vibration frequency as a function of the molecular weight on their surface using the experimental measurements, and the ability of these calibrated nanobalances is demonstrated by studying the relative binding affinities of different thiol ligands at the NPL surface.

\section{Results}

\section{Nanobalance calibration}

In order to characterize the sensitivity of the breathing vibration of the NPLs to the mass of the ligands present on its surface, we prepared CdSe NPLs with a 5 monolayer thickness. After synthesis, the surface of the NPLs is covered with oleic acid ligands (OA, molecular weight: $282.46 \mathrm{~g} \mathrm{~mol}^{-1}$ ) and the black curves in Fig. $1 \mathrm{a}$ and $\mathrm{b}$ show the corresponding
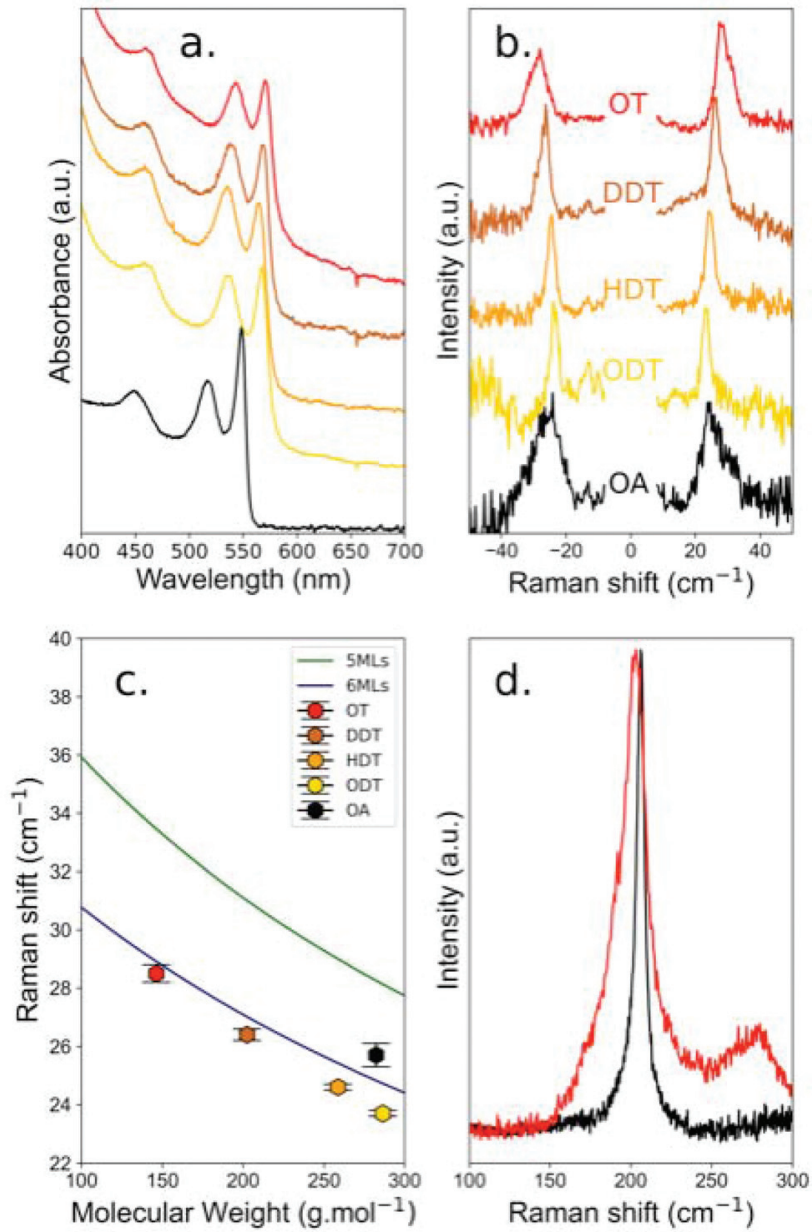

Fig. 1 (a) Optical absorption spectra of 5 ML NPLs as a function of the ligand type (from top to bottom: OT, DDT, HDT, ODT and OA). (b) Corresponding low frequency Raman spectra (the central line corresponding to the Rayleigh scattering has been removed for clarity). (c) Comparison of the experimental frequency with the mass load model. ${ }^{15}$ (d) Raman spectra of NPLs with OA ligands (black) and NPLs with OT ligands (red). 
UV-Visible absorption and low frequency Raman spectra respectively. The initial absorption spectrum shows two peaks at 515 and $550 \mathrm{~nm}$ that are the typical signature of the exciton confinement in a 5 monolayer NPL with oleic acid ligands. ${ }^{23}$ After ligand exchange by alkyl-thiols (see Material and methods), the excitonic energy is reduced, which results in a red shift of the exciton peaks to 536 and $567 \mathrm{~nm}$ respectively, confirming that the ligand exchange is complete. ${ }^{20,24}$ The length of the thiol chain, varied to modify the mass of the ligands from $146.29 \mathrm{~g} \mathrm{~mol}^{-1}$ for octanethiol (OT), to $286.56 \mathrm{~g}$ $\mathrm{mol}^{-1}$ for octadecanethiol (ODT), does not influence significantly the energy of the exciton. To measure the vibration frequency as a function of the ligand molecular weight, the measurements must be performed in close resonance with the lower energy exciton peak. ${ }^{15}$ Under such a condition, a strong luminescence background overwhelming the Raman signal is observed, and a low concentration of copper ions is introduced into the NPLs in order to quench a part of the luminescence (see the Methods section).

Fig. 1b shows the variation of the breathing vibration frequency as a function of the thiol ligand molecular weight. An increase in the ligand molecular weight induces a down-shift in the frequency (from $28.7 \mathrm{~cm}^{-1}$ to $23.5 \mathrm{~cm}^{-1}$ ), as predicted in our previous work. ${ }^{15}$ However, the vibration frequencies measured for OA and ODT whose masses are almost the same (see the Methods section), are significantly different: $25.7 \mathrm{~cm}^{-1}$ and $23.5 \mathrm{~cm}^{-1}$ respectively. This behavior is reported in Fig. 1c, where the experimental results (hexagonal dots) are compared with those of the mass-load model ${ }^{15}$ for 5 monolayer and 6 monolayer thick NPLs. Surprisingly, the measured frequency shifts as a function of thiol molecular weights are in good agreement with the model only if we consider 6 ML NPLs, while they are in between 5 and 6 monolayers for OA ligands.

The influence of thiol ligands at the surface of CdSe nanocrystals has already been investigated. This type of ligand induces a red-shift of the lowest excitonic transition of CdSe nanocrystals, ${ }^{25-27}$ as shown in Fig. 1a for our NPLs. This shift has first been interpreted as a decrease in the quantum confinement of the hole due to the presence of the ligands. ${ }^{25-27}$ However, this effect can also be explained if one considers that the sulfur atoms of the thiol ligands are forming a shell around the CdSe nanoplatelets, thus increasing slightly its effective thickness. Then, it is the delocalization of the exciton that induces a red-shift in the excitonic peak. ${ }^{5}$ In the case of NPLs, considering that the sulfur atoms coming from the thiol ligands are part of the lattice structure, half a monolayer is added on each side of the NPLs, increasing the total thickness by one monolayer. If we consider this hypothesis with the mass load model, the agreement with the experimental measurements is significantly improved for thiol molecules as shown in Fig. 1c. The presence of this sulfide layer is supported by the Raman spectra at higher frequency (between 200 and $300 \mathrm{~cm}^{-1}$ ) where the longitudinal optical phonons of CdS and CdSe manifest. Fig. 1d shows the Raman spectrum measured on the sample of NPLs covered with OT ligands. In this spectrum, an intense asymmetric peak at $203 \mathrm{~cm}^{-1}$ and a band at around $275 \mathrm{~cm}^{-1}$ are observed. The first peak corresponds to the CdSe LO phonon. The broadening of the peak with respect to the bulk CdSe LO phonon is explained by the increasing role of surface optical phonons (at lower frequency) and by the spatial confinement that induces scattering from phonons (higher frequency). ${ }^{28-30}$ The second band has already been observed ${ }^{5,24,29-32}$ when performing resonant Raman measurements on CdSe QDs plus CdS or elemental S. It was assigned to the presence of sulfur atoms that form CdS bonds whose LO phonon frequency depends on the thickness of the CdS layer. For a single monolayer, the frequency of this CdSlike LO phonon is around $270 \mathrm{~cm}^{-1}$. The difference in frequency between the CdSe LO phonon and the CdS-like LO phonon can be used to estimate the quantity of Se atoms with respect to $\mathrm{S}$ atoms. ${ }^{33}$ Here, a difference of $72 \mathrm{~cm}^{-1}$ is measured, which approximately corresponds to $80 \%$ of Se and $20 \%$ of S atoms. This is the expected ratio if we consider NPLS of 5 MLs with half monolayer of sulfur on each side. Unfortunately, this band was not observed on all samples due to the luminescence background, but the effect of the sulfur bonds is also clearly evidenced at low frequency when considering the breathing vibration mode of the NPLs.

Indeed, the origin of this vibration mode at low frequency has also been discussed by A. I. Lebedev in ref. 34. In this publication, the author uses first-principles calculations and modeling of the Raman spectra to determine the origin of this vibrational peak. CdSe NPLs of different thicknesses (2 to 6 MLs) are modeled with a fluorine atom layer compensating the cadmium atom surface charge for the calculations. Vibration modes with a displacement out of plane, qualified as quasiLamb, are calculated, but their frequencies are not in good agreement with our experimental frequencies for NPLs covered with OA. However, it is interesting to note that fluorine atoms are placed in the vacant selenium positions, and that the wave confinement calculated in 34 is defined based on the thickness of the plate plus the two layers of fluorine atoms. These theoretical frequencies are therefore obtained for a bare platelet of CdSe with an additional layer of fluorine atoms and must be compared to the Lamb model of a bare NPL with the same thickness. When considering this additional monolayer, a good agreement between the approach in ref. 34 and the Lamb model is observed. These results thus confirm that the sulfur atom constituting the thiol group is involved in a molecular bond with the cadmium atoms present at the surface of the NPLs.

This influence of the binding group explains why the NPLS with OA ligands do not follow the same behavior as that of the NPLs with thiol ligands. However, in the case of OA, there is still a discrepancy between the mass-load model for 5 monolayers and the experimental measurement.

Due to the preparation method, the NPL surface is almost completely covered with carboxylates, ${ }^{35}$ that is a mixture of oleate and acetate ligands (in unknown proportions). The overall molecular weight to be taken into account should then be lower and may lead to a better agreement with that of the 
mass-load model. Moreover, one could expect that the oxygen atoms of the carboxylic function of OA might also be involved in the structure of the NPLs. To understand this behavior, thinner NPLs with thicknesses of 3 and 4 monolayers were synthesized with OA native ligands, and we carried out the same study with thiol ligand exchange.

The measured frequencies of the breathing mode are reported in Fig. 2 and compared with those of the mass-load model. The data are represented as a function of the molecular weight of the ligands (Fig. 2a) and as a function of the number of layers for fixed molecular weight (Fig. 2b and c). As demonstrated previously, with thiols we have to consider that the thickness increases by one monolayer and thus if we compare NPLs with the same thicknesses we observe that the frequency shifts measured for OA and ODT are almost the same. This confirms that the sulfur atoms are part of the lattice structure of the NPLs.

It is clear from Fig. 2 that the increase of the thiol length, i.e. the surface mass experienced by the NPLs, induces, as expected, a decrease of the breathing vibration mode frequency for all the thicknesses considered. Nevertheless, the massload model only reproduces correctly the behavior observed for 6 ML NPLs. When considering 5 ML NPLs, the agreement is still good, but a deviation from the model is observed for the heaviest molecules. When reducing the thick- ness to 4 MLs, the discrepancy between our model and the experimental data increases again. Fig. $2 b$ and $c$ represent the same set of data as a function of the inverse thickness of the NPLs and are compared with the ideal case of a bare NPL. For the thiol molecules, we clearly observe that the measured frequencies are lower than the frequency expected with the massload model. The difference between these frequencies increases when the molecular weight of the thiols increases, and this effect is all the greater as the NPL thickness is reduced. Fig. 2c also shows a comparison between ODT and OA molecules that have almost the same molecular weight. Albeit the general behavior is the same (experimental frequencies lower than expected frequencies and function of the NPL inverse thickness), the experimental frequencies for OA are closer to those of the mass-load model and follows a different behavior than those for ODT. These observations suggest that the mass-load model is still incomplete for thin NPLs.

This model is based on several hypotheses: the first one is the surface coverage. To calculate the surface mass density of the ligands at the surface of the NPLs, we considered that each surface cadmium atom is linked to one ligand (surface density: 5.4 ligands per $\mathrm{nm}^{2}$ ). This is an overestimation because the typical values deduced by NMR are between 5.3 and 4.6 ligands per $\mathrm{nm}^{2}$ for OA ligands. ${ }^{35,36}$ The effect of a lower surface covering results in an increase in the frequency
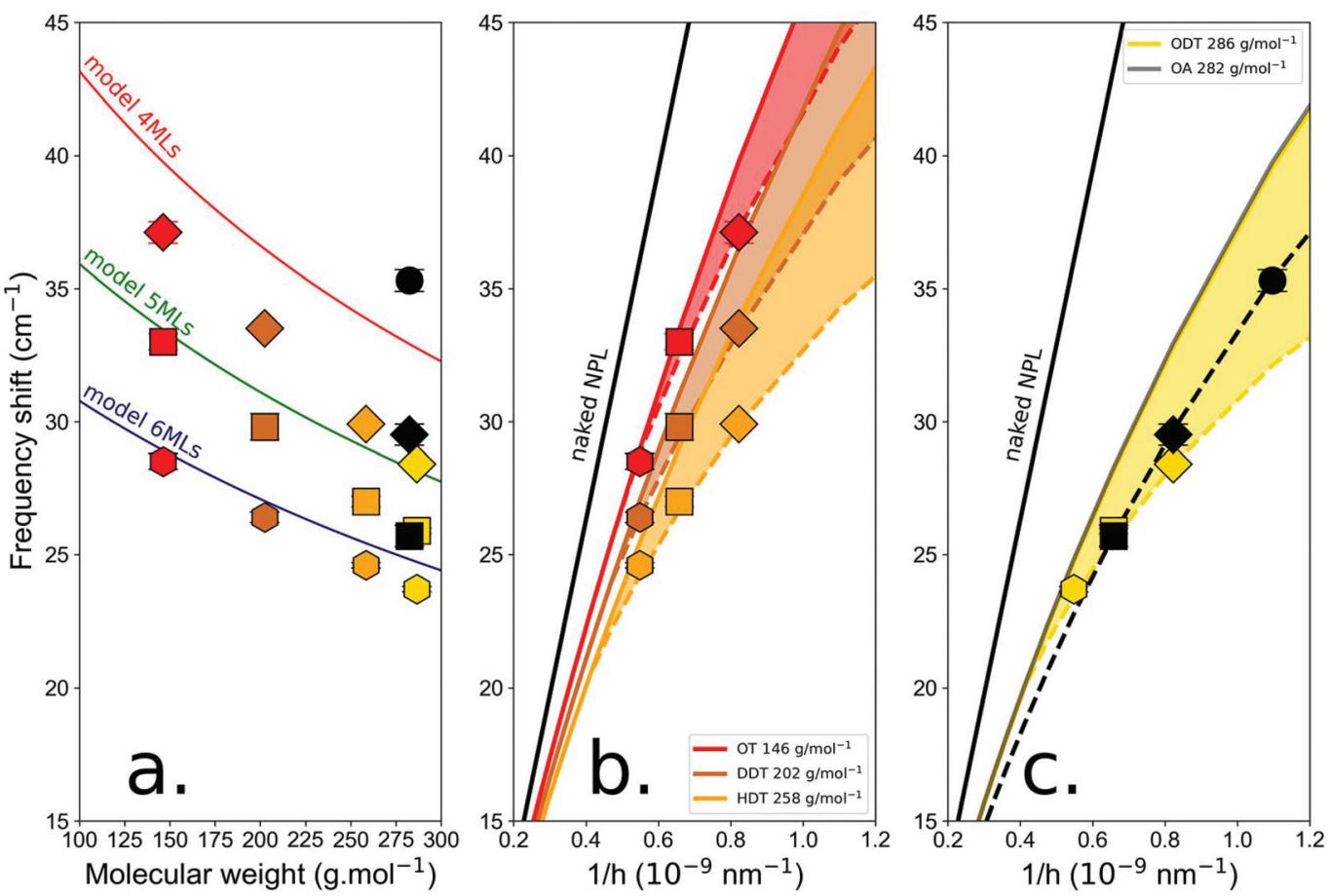

Fig. 2 (a) Measured frequencies of NPL vibration modes as a function of thiol molecular weight for different NPL thicknesses. Vibration frequencies of the initial oleate NPLs are also included. Red, brown, orange, yellow and black symbols correspond to OT, DDT, HDT, ODT, and OA respectively. Hexagon, square, diamond, and circle symbols correspond to 6, 5, 4, and $3 \mathrm{ML}$ thick. The experimental data are compared with the mass load model $^{15}$ for the different thicknesses: 6 MLs (blue line), 5 MLs (green line), and 4 MLs (red line). (b) and (c) Data for OT (red), DDT (brown), HDT (orange), ODT (yellow) and OA (black) are represented as functions of the inverse thickness of the NPLs and compared to the theoretical value for a naked NPL (black curve) (figures (b) and (c) are splitted for clarity). Continuous lines are calculated using the mass-load model. The dotted lines are guide to the eye, that are obtained by considering a modification of the sound longitudinal sound velocity that depends on the molecular weight. 
shifts (Fig. S1 of the ESI $\dagger$ ) which is not the experimental behavior. One would expect a different surface covering for thiol ligands, but thiol molecules have a greater affinity with nanoplatelets than OA, and therefore one can expect at least the same surface covering. Finally, while the surface covering of CdSe nanoplatelets with thiol molecules has not been measured, the surface covering of thiol molecules on gold surfaces has been extensively studied showing that the alkane chain length does not affect the covering. ${ }^{37-39}$ These works also highlight the fact that the geometric effect can induce an increase in surface covering. In what follows, a fixed surface density of 5.4 ligands per $\mathrm{nm}^{2}$ is therefore considered whatever the nature of the ligand.

The other parameter that can be modified is the value of the sound velocity of the CdSe nanoplatelet, that is related to its elastic and structural properties. In our model, we used the bulk value of CdSe, considering the zinc-blende structure of the NPLs: the lattice parameter $a=0.608 \mathrm{~nm}$ and the theoretically estimated elastic constant $C=C_{11}=88 \mathrm{GPa}$, giving a longitudinal sound velocity of $3945 \mathrm{~m} \mathrm{~s}^{-1} \cdot{ }^{15,16}$ These values were deduced from the bulk material considering a zincblende structure, but it is an estimation. In particular, several works have shown that the crystalline structure of NPLs is slightly different from the zinc-blende structure. ${ }^{21,40}$
The TEM images (Fig. 3) of the NPLs clearly show that ligands strongly impact their morphology. They induce a winding of the NPLs whose amplitude varies with the thiol ligand length. This behavior is unexpected and might be related to steric hindrance. Moreover, the nature of the bond between the molecule and the surface is also probably important to control the winding, but further experiments with different ligands of well-known steric hindrance and functional type should be performed to answer more specifically this question. Be it as it may, the TEM images show a clear dependency of the winding radius as a function of the thiol length.

We performed PXRD measurements on our samples to determine the in-plane and out-of-plane lattice parameters (diffractograms in the ESI $\dagger$ ). Fig. 3a confirms that the crystalline structure of the NPLs is more accurately described as pseudotetragonal, due to the differences between in-plane and out-of-plane lattice parameters. ${ }^{40}$ The measured in-plane lattice parameter is slightly smaller $(1 \%)$ than the out-of-plane parameter. Moreover, the in-plane and out-of-plane lattice parameters decrease slightly as a function of the alkyl chain length. It has to be noticed that due to the nano-2D nature of the sample, the peaks observed on the PXRD diffractograms and corresponding to the out-of-plane lattice parameter are
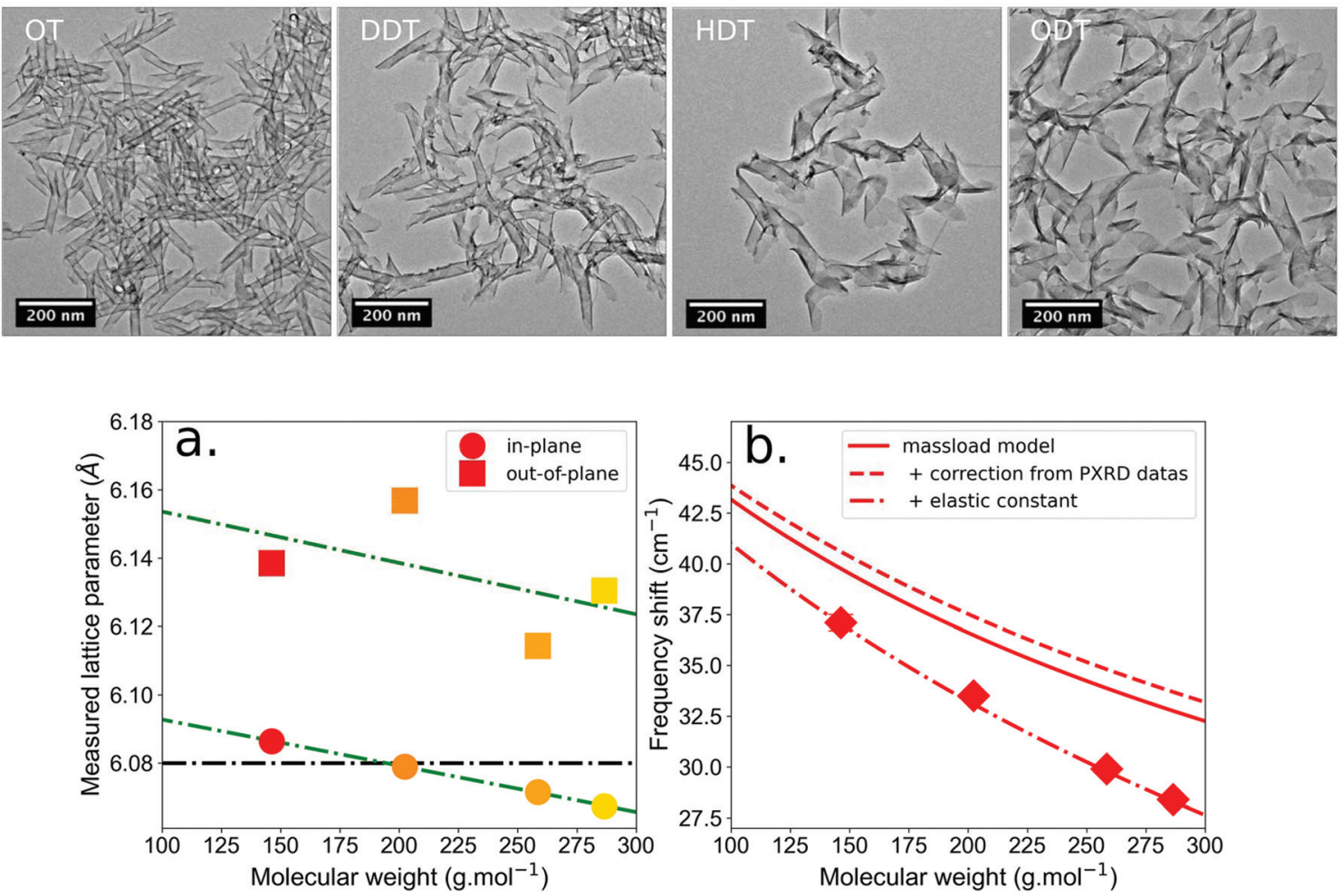

Fig. 3 Top. TEM images of the NPL ( $3+1 \mathrm{MLs})$ morphology as a function of the ligand. (a) In-plane and out-of-plane lattice parameters measured by XRD for the 4 monolayer NPLs as a function of the molecular weight of the ligands. (b). The continuous red line corresponds to the mass load model considering a zinc-blende structure with a constant $C=88 \mathrm{GPa}$ and the bulk lattice parameter $a=6.08$ angstroms. The dotted red line considers the modification of the lattice parameter as a function of the ligand mass deduced from PXRD measurements, and the induced modification of the density $\rho$. Finally, the dashed red line considers an additional modification of the elastic constant $C$ depending on the ligand molecular weight. This latter curve is used as a calibration curve of the nanobalances with 4 ML thickness. 
difficult to fit and more precise PXRD measurements may be needed to refine the accuracy of the in plane and out of plane lattice parameters. Yet, Fig. 3a thus confirms that a modification of the lattice parameters and thus of the unit cell depends on the thiol chain length. This structure modification, from cubic to tetragonal, implies to take $C_{33}$ as the elastic parameter along the $c$-axis of the NPLs (i.e. along the thickness), instead of $C_{11}$. Unfortunately, the elasticity of such a structure at the nanoscale is poorly characterized and no value of $C_{33}$ is reported, a fortiori as a function of ligand mass. Therefore, we rely on an empirical dependence of $C_{33}$ as a function of ligand mass to calibrate our nanobalances. Thus, by fitting the experimental data with our mass load model considering the modification of the lattice parameter (and density $\rho$ ) (see the ESI $\dagger$ ) we can deduce, for the 4 ML NPLs, the dependency of the elastic parameter as a function of the ligand mass $C_{33}=C-0.091 \times M$; where $C=C_{11, \mathrm{ZB}}=88 \mathrm{GPa}$ and $M$ is the molecular weight in $\mathrm{g} \mathrm{mol}^{-1}$. This dependency is used in Fig. $3 \mathrm{~b}$ to calibrate the behavior of our nanobalances. Although the linear dependency of the elastic parameter as a function of the unit cell deformation (and thus on the molecular weight) is expected, $C_{33, \mathrm{ZB}}$ should be replaced by the value of the elastic constant of a bare NPL of 4 MLs. Unfortunately, this value is not yet available. In addition, this dependence is extracted from the experimental data considering a surface covering of $100 \%$. This is a strong assumption which also means that the potential structural deformation of NPLs is potentially greater than we expected. Anyway, the nanobalances are simply calibrated using the experimental data and the unique unknown quantity is the surface covering which is considered constant regardless of the nature of the ligands to determine the difference in binding affinity between OT and ODT.

\section{Using the nanobalance effect to monitor the binding of ligands}

In the previous part of the article, the study of the frequency shifts gave us insight into mechanical property variations of the nanoplatelets grafted with ligands of different chain lengths and binding groups that must be considered to calibrate the nanobalances. It has been possible to obtain an experimental relationship linking the frequency vibration of the NPL to the molecular weight of the ligands attached to the surface in the case of linear alkanethiols. We will now explore the possibility to use the NPL as a nanobalance that can allow the precise analysis of the composition of surface ligands in more complex cases where other techniques are not sensitive.

As we establish this relationship for linear alkanethiols, we will consider the following equilibrium of binding between octanethiol and octadecanethiol at the surface of the nanoplatelets:

$$
\begin{aligned}
& \mathrm{Cd}_{\mathrm{NPL}}-\text { octadecanethiolate }+\mathrm{OT} \\
& \quad \rightleftharpoons \mathrm{Cd}_{\mathrm{NPL}}-\text { octanethiolate }+\mathrm{ODT}
\end{aligned}
$$

The equilibrium constant $K$ is simply expressed by:

$$
K_{\mathrm{NPL}-\text { thiols }}=\frac{[\mathrm{ODT}]\left[\mathrm{Cd}_{\mathrm{NPL}} \mathrm{OT}\right]}{[\mathrm{OT}]\left[\mathrm{Cd}_{\mathrm{NPL}} \mathrm{ODT}\right]}
$$

We are expecting $K$ to be near a value of 1 , as the differences between the two considered ligands only reside in their chain length that shouldn't greatly modify their affinity towards the NPL cadmium surface cations. The experiment simply consists of mixing 3 ML NPLs bearing their native carboxylate ligands with a large excess of an ODT : OT binary mixture of varying ratios. The measured spectra are shown in the ESI (Fig. S4 $\dagger$ ) and Fig. 4 shows the measured frequencies as a function of the molecular weight and ODT fraction. The ligand exchange is allowed to proceed at $65{ }^{\circ} \mathrm{C}$ for $72 \mathrm{~h}$ as described in the Experimental section. Considering that the thiols are in large excess compared to the native carboxylates and that their affinity for the NPL surface is much higher, the observed equilibrium is practically the one noted above. If we call $x$ the fraction of ODT in solution and $y$ the fraction of ODT at the surface of the NPLs, the equilibrium can be written as:

$$
K_{\mathrm{NPL}-\text { thiols }}=\frac{[\mathrm{ODT}]\left[\mathrm{Cd}_{\mathrm{NPL}} \mathrm{OT}\right]}{[\mathrm{OT}]\left[\mathrm{Cd}_{\mathrm{NPL}} \mathrm{ODT}\right]}=\frac{x}{(1-x)} \times \frac{(1-y)}{y}
$$

Considering that the excess of free ligands is very large, $y$ is then determined using the low frequency Raman measurement followed by the calculation of the average molar mass of ligands needed to reach this frequency according to the calibration curve (Fig. 3b). The results presented in Fig. 4 show a clear deviation to the linear case ( $K=1$ green line), and the experimental values can be fitted reasonably well using our simple equilibrium model with $K=2.7$. This simple competitive binding experiment unambiguously demonstrates that OT has a higher affinity than ODT for the NPL surface cadmium ions and allows us to quantify the affinity difference. This result also allows the determination of the energy difference

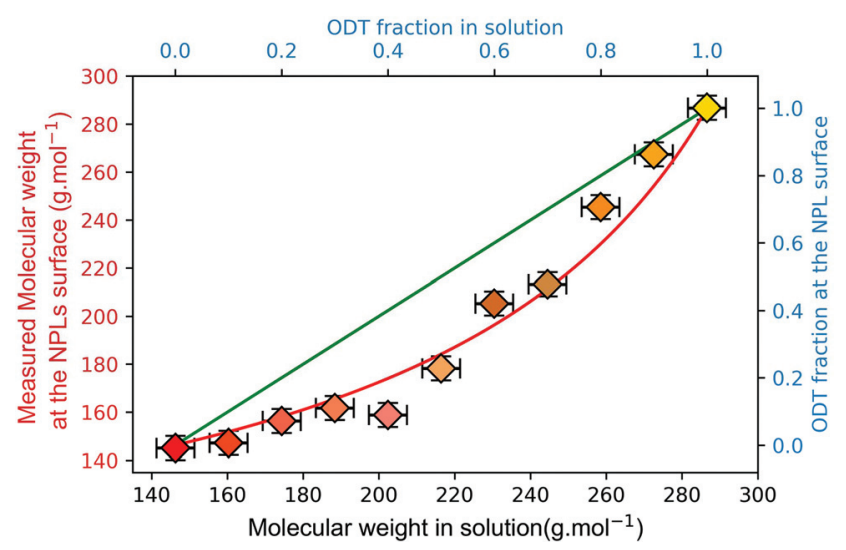

Fig. 4 Competitive binding between octanethiol and octadecanethiol on $3 \mathrm{ML}$ CdSe NPLs using frequency shift measurement to determine the weight of the surface-bound ligands. The green and red curves are the result of the equilibrium model considering $K=1$ and $K=2.7$ respectively. 
between Cd-OT and Cd-ODT molecular bonds at the NPL surface. If we consider that:

$$
K_{\mathrm{d}(\mathrm{OT})}=C \times e^{\left(\Delta G_{\mathrm{OT}-\mathrm{NPL}} / \mathrm{RT}\right)}
$$

It is possible to determine the energy difference $\Delta G_{\mathrm{OT}-\mathrm{NPL}}-$ $\Delta G_{\text {ODT-NPL }}$ since:

$$
\begin{aligned}
K_{\mathrm{NPL}-\text { thiols }} & =\frac{[\mathrm{ODT}]\left[\mathrm{Cd}_{\mathrm{NPL}} \mathrm{OT}\right]}{[\mathrm{OT}]\left[\mathrm{Cd}_{\mathrm{NPL}} \mathrm{ODT}\right]}=\frac{K_{\mathrm{d}(\mathrm{OT})}}{K_{\mathrm{d}(\mathrm{ODT})}} \\
& =\frac{e^{\left(\Delta G_{\mathrm{OT}-\mathrm{NPL}} / R T\right)}}{e^{\left(\Delta G_{\mathrm{ODT}-\mathrm{NPL}} / R T\right)}}=e^{\frac{1}{R T}\left(\Delta G_{\mathrm{OT}-\mathrm{NPL}}-\Delta G_{\mathrm{ODT}-\mathrm{NPL}}\right)}
\end{aligned}
$$

We measured $K_{\text {NPL-thiols }}=2.7$ which gives an energy difference of $\Delta G_{\text {OT-NPL }}-\Delta G_{\text {ODT-NPL }} \approx R T=2.48 \mathrm{~kJ} \mathrm{~mol}^{-1}$.

These results clearly show that the vibrations of nanoplatelets are a really sensitive signature of their structure as well as their surface ligands. Being able to disentangle these effects, it gives access to a sensitive way to probe their mechanical properties and to follow their surface modifications.

In conclusion, we have here demonstrated the sensitivity of the breathing vibration mode of CdSe NPLs to the molecular weight of ligands. By carefully considering the real thickness of the NPLs, we highlight that for relatively thick (6 MLs) NPLs, this breathing mode depends on the molecular weight of ligands and follows the behavior predicted by the mass-load model we previously developed. However, when reducing the number of layers to potentially increase the breathing mode sensitivity to the weight variations of ligands, we observed that other effects must be considered. At this scale, the structure of the whole NPL is impacted by the surface modifications induced by the ligands. This results in a modification of the NPL mechanical properties that strongly depends on the nature of the ligands. To understand the dependency of the mechanical properties of the NPL plus the ligand system, further experimental and theoretical investigations must be performed. Nevertheless, we were able to calibrate the nanobalances based on the relationship between the NPL vibration frequency and the molecular weight of ligands of the same type (alkyl chains), which was deduced experimentally. This calibration has finally been applied to monitor the competitive binding at the surface of 3 ML NPLs between thiols of different chain lengths. Although the development of more comprehensive models is still needed, the ability to obtain information on the structural properties by simply using inelastic light scattering spectroscopy opens new routes to investigate the physical-chemistry of the interfaces, and the effect of surface treatments on nano-objects. Finally, these results open a route to develop a new class of label-free sensors based on a nanoplatelet acting as a nanobalance probed through inelastic light scattering.

\section{Methods}

\section{Chemicals}

1-Octadecene (ODE, 90\%), oleic acid (90\%) and 1-octylamine (99\%) were purchased from Alfa Aesar. 1-Octanethiol (OT,
98.5\%), 1-octadecanethiol (ODT, 98\%), 1-dodecanethiol (DDT, 98\%), 1-hexadecanethiol (HDT, 95\%), trioctylphosphine (TOP, 97\%), tetrakis(acetonitrile)copper(I) hexafluorophosphate (97\%), cadmium nitrate tetrahydrate $\left(\mathrm{Cd}\left(\mathrm{NO}_{3}\right) \cdot 4 \mathrm{H}_{2} \mathrm{O}, 99 \%\right)$ and cadmium acetate dihydrate (98\%) were obtained from Sigma-Aldrich. Selenium powder (99.99\%) was purchased from Strem. $\mathrm{Na}$ (myristate) (98\%) was purchased from TCI.

All chemicals were used directly without purification.

\section{Precursors}

Cd(myristate)2. $9.3 \mathrm{~g}$ of $\mathrm{Na}$ (myristate) is dissolved in methanol under vigorous magnetic stirring for $1 \mathrm{~h} .3 .7 \mathrm{~g}$ of $\mathrm{Cd}$ $\left(\mathrm{NO}_{3}\right) \cdot 4 \mathrm{H}_{2} \mathrm{O}$ dissolved in $120 \mathrm{~mL}$ of $\mathrm{MeOH}$ is slowly added to this solution inducing $\mathrm{Cd}(\mathrm{myr})_{2}$ precipitation. After stirring for one more hour, the white solid is filtered and washed multiple times on a Buchner funnel.

\section{Synthesis of ML CdSe NPLs}

$14 \mathrm{~mL}$ of ODE, $185 \mathrm{mg}$ of $\mathrm{Cd}($ acetate $) 2 \cdot 2 \mathrm{H}_{2} \mathrm{O}$ and $190 \mu \mathrm{L}$ of oleic acid are introduced in a $100 \mathrm{~mL}$ three neck flask. The temperature of the solution is raised to $220^{\circ} \mathrm{C}$ under an argon flux. After injection of $300 \mu \mathrm{L}$ of $1 \mathrm{M}$ TOPSe, the reaction mixture is annealed at the same temperature for 10 minutes resulting in the formation of $3 \mathrm{ML}$ CdSe NPLs. At the end of the reaction, $2 \mathrm{~mL}$ of oleic acid is added before the solution is allowed to cool to room temperature. NPLs are isolated from the reaction mixture by centrifugation; they are then dispersed in hexane and precipitated one more time by addition of ethanol. The final product is dispersed in $10 \mathrm{~mL}$ of hexane for storage.

\section{Synthesis of ML CdSe NPLs}

$85 \mathrm{~mL}$ of $\mathrm{ODE}$ and $1.02 \mathrm{~g}$ of $\mathrm{Cd}(\mathrm{myr})_{2}$ are introduced in a $250 \mathrm{~mL}$ three-neck flask and degassed $1 \mathrm{~h}$ under a vacuum at $100{ }^{\circ} \mathrm{C}$. Under argon, $72 \mathrm{mg}$ of Se powder dispersed in $5 \mathrm{~mL}$ of ODE is introduced in the flask. The temperature of the setup is set to $240{ }^{\circ} \mathrm{C}$ and $300 \mathrm{mg}$ of $\mathrm{Cd}$ (acetate) $)_{2}$ is introduced in the flask at $205{ }^{\circ} \mathrm{C}$. The reaction mixture is annealed at $240{ }^{\circ} \mathrm{C}$ for 12 minutes resulting in the formation of $4 \mathrm{ML}$ CdSe NPLs. At the end of the reaction, $6 \mathrm{~mL}$ of oleic acid is added before the solution is allowed to cool to room temperature. NPLs are isolated from the reaction mixture by precipitation using a hexane/ethanol mixture and centrifugation; they are then dispersed in hexane and precipitated one more time by addition of ethanol. The final product is dispersed in hexane for storage, adjusting the solution volume to obtain an optical density of 1.5 at $350 \mathrm{~nm}$ when diluted 15 times.

\section{Synthesis of ML CdSe NPLS}

$56 \mathrm{~mL}$ of $\mathrm{ODE}$ and $680 \mathrm{mg}$ of $\mathrm{Cd}(\mathrm{myr})_{2}$ are introduced in a $250 \mathrm{~mL}$ three-neck flask and degassed $1 \mathrm{~h}$ under a vacuum at $100{ }^{\circ} \mathrm{C}$. The temperature of the solution is raised to $250{ }^{\circ} \mathrm{C}$ under an argon flux and $48 \mathrm{mg}$ of Se powder dispersed in 2 ML ODE is swiftly injected at this temperature. Immediately after injection, $480 \mathrm{mg}$ of $\mathrm{Cd}$ (acetate) $)_{2}$ is introduced in the 
flask. The reaction mixture is annealed at the same temperature for 15 minutes resulting in the formation of $5 \mathrm{ML}$ CdSe NPLs. At the end of the reaction, $10 \mathrm{~mL}$ of oleic acid is added before the solution is allowed to cool to room temperature. NPLs are isolated from the reaction mixture by centrifugation; they are then dispersed in hexane and precipitated one more time by addition of ethanol. The final product is dispersed in hexane for storage, adjusting the solution volume to obtain an optical density of 1.5 at $350 \mathrm{~nm}$ when diluted 15 times.

\section{Ligand exchange}

The ligand exchange protocol is adapted from ref. 20 . Typically, $200 \mu \mathrm{L}$ of the as-synthetized NPLs in hexane are mixed with $2 \mathrm{~mL}$ of hexane and $1 \mathrm{mmol}$ alkanethiol (octanethiol, dodecanethiol, hexadecanethiol or octadecanethiol). The mixture is then placed in an oven at $65{ }^{\circ} \mathrm{C}$ for $72 \mathrm{~h}$ to allow for a complete ligand exchange to occur. After exchange, the NPLs are washed trice by precipitation with ethanol, centrifugation and dispersion by sonication in $5 \mathrm{~mL}$ of hexane.

\section{Copper doping}

A stock solution of tetrakis(acetonitrile)copper(I) hexafluorophosphate, $[\mathrm{MeCN}]_{4} \mathrm{Cu}^{\mathrm{I}} \mathrm{PF}_{6}$, at $1 \times 10^{-4} \mathrm{M}$ in ethanol is used to dope the NPLs. The stock solution of copper(I) ions is directly added to NPLs in hexane at a ratio of $1 \mathrm{Cu}$ for $1000 \mathrm{Cd}$, and the mixture is heated at $65^{\circ} \mathrm{C}$ for $1 \mathrm{~h}$. Excess ions are then discarded by precipitation in ethanol, and by centrifugation and dispersion in hexane. This copper doping allows the quenching of part of the luminescence in order to observe the Raman scattering signal.

Absorbance spectra. Absorbance spectra have been recorded using an AvaSpec-ULS2048 fiber optic spectrometer equipped with an AvaLight balanced deuterium-halogen lightsource.

PXRD. Powder X-ray diffraction (PXRD) patterns were recorded on a PANalytical Empyrean diffractometer ( $\mathrm{Cu} \mathrm{K} \alpha$ radiation). The samples were prepared by drying on a low background silicon substrate.

Raman spectroscopy. Resonant Raman spectra were recorded using Labram $\mathrm{HR}$ and Renishaw microspectrometers equipped with ultra-low frequency notch filters. The spectra were acquired using different laser lines (532, 633, and $660 \mathrm{~nm}$ ) depending on the thicknesses of the NPLs and thus on the energy of the excitons. A long working distance $\times 50$ objective was used and each time the laser power was fixed to avoid any damage on the sample for several hours (typical: $10 \mathrm{~mW}$ ). Several spectra were acquired at different positions of the same samples and were used to extract the frequencies using a gaussian fitting method.

\section{Conflicts of interest}

There are no conflicts to declare.

\section{Acknowledgements}

This work was partially supported by the ANR NanoVip project, grant ANR.13.JS10.0002 of the French Agence National de la Recherche, the Fédération André Marie Ampère 2013 (FRAMA) and the Programme Avenir Lyon Saint-Etienne from Université de Lyon in the framework "Investissements d'Avenir" (ANR-11-IDEX-0007). L. S. acknowledges support from EUR-EIPHI Graduate School (contract ANR-17EURE-0002). The authors declare no competing financial interest. We thank the Consortium Lyon St-Etienne de Microscopie (CLYM) for access to the microscope platform TEM characterization, and the spectroscopy platform CECOMO at Villeurbanne for the access to the LABRAM-HR spectrometer.

\section{References}

1 A. M. Kelley, Q. Dai, Z. Jiang, J. A. Baker and D. F. Kelley, Resonance Raman spectra of wurtzite and zincblende CdSe nanocrystals, Chem. Phys., 2013, 422, 272-276.

2 J. A. Baker, D. F. Kelley and A. M. Kelley, Resonance Raman and photoluminescence excitation profiles and excitedstate dynamics in CdSe nanocrystals, J. Chem. Phys., 2013, 139, 024702.

3 C. Lin, D. F. Kelley, M. Rico and A. M. Kelley, The "Surface Optical" Phonon in CdSe Nanocrystals, ACS Nano, 2014, 8, 3928-3938.

4 C. Lin, K. Gong, D. F. Kelley and A. M. Kelley, ElectronPhonon Coupling in CdSe/CdS Core/Shell Quantum Dots, ACS Nano, 2015, 9, 8131-8141.

5 J. J. Grenland, C. Lin, K. Gong, D. F. Kelley and A. M. Kelley, Resonance Raman Investigation of the Interaction between Aromatic Dithiocarbamate Ligands and CdSe Quantum Dots, J. Phys. Chem. C, 2017, 121, 7056-7061.

6 A. J. Mork, E. M. Y. Lee and W. A. Tisdale, Temperature dependence of acoustic vibrations of CdSe and CdSe-CdS core-shell nanocrystals measured by low-frequency Raman spectroscopy, Phys. Chem. Chem. Phys., 2016, 18, 2879728801.

7 C. J. A. Maddux, D. F. Kelley and A. M. Kelley, Weak Exciton-Phonon Coupling in CdSe Nanoplatelets from Quantitative Resonance Raman Intensity Analysis, J. Phys. Chem. C, 2018, 122, 27100-27106.

8 V. Paillard, et al., Strain characterization of strained silicon on insulator wafers, Microelectron. Eng., 2004, 72, 367-373.

9 M. Cazayous, et al., Strain and composition in selfassembled SiGe islands by Raman spectroscopy, J. Appl. Phys., 2002, 91, 6772.

10 M. Miscuglio, M. L. Lin, F. Di Stasio, P. H. Tan and R. Krahne, Confined Acoustic Phonons in Colloidal Nanorod Heterostructures Investigated by Nonresonant Raman Spectroscopy and Finite Elements Simulations, Nano Lett., 2016, 16, 7664-7670.

11 P. Mukherjee, S. J. Lim, T. P. Wrobel, R. Bhargava and A. M. Smith, Measuring and Predicting the Internal 
Structure of Semiconductor Nanocrystals through Raman Spectroscopy, J. Am. Chem. Soc., 2016, 138, 10887-10896.

12 H. Lamb, On the Vibrations of an Elastic Sphere, Proc. London Math. Soc., 1881, s1-13, 189-212.

13 A. J. Mork, E. M. Y. Lee, N. S. Dahod, A. P. Willard and W. A. Tisdale, Modulation of Low-Frequency Acoustic Vibrations in Semiconductor Nanocrystals through Choice of Surface Ligand, J. Phys. Chem. Lett., 2016, 7, 4213-4216.

14 D. P. Morgan, C. J. A. Maddux and D. F. Kelley, Transient Absorption Spectroscopy of CdSe Nanoplatelets, J. Phys. Chem. C, 2018, 122, 23772-23779.

15 A. Girard, et al., The mass load effect on the resonant acoustic frequencies of colloidal semiconductor nanoplatelets, Nanoscale, 2016, 8, 13251-13256.

16 A. Girard, et al., Environmental effects on the natural vibrations of nanoplatelets: a high pressure study, Nanoscale, 2017, 9, 6551-6557.

17 C. L. Poyser, et al., Coherent Acoustic Phonons in Colloidal Semiconductor Nanocrystal Superlattices, ACS Nano, 2016, 10, 1163-1169.

18 Q. Martinet, et al., Performances of the Lamb Model to Describe the Vibrations of Gold Quantum-Sized Clusters, J. Phys. Chem. C, 2020, 124, 19324-19332.

19 G. Bachelier, J. Margueritat, A. Mlayah, J. Gonzalo and C. N. Afonso, Size dispersion effects on the low-frequency Raman scattering of quasispherical silver nanoparticles: Experiment and theory, Phys. Rev. B: Condens. Matter Mater. Phys., 2007, 76, 235419.

20 B. Mahler, B. Nadal, C. Bouet, G. Patriarche and B. Dubertret, Core/Shell Colloidal Semiconductor Nanoplatelets, J. Am. Chem. Soc., 2012, 134, 1859118598.

21 A. Antanovich, et al., A strain-induced exciton transition energy shift in CdSe nanoplatelets: the impact of an organic ligand shell, Nanoscale, 2017, 9, 18042-18053.

22 R. B. Vasiliev, et al., Spontaneous Folding of CdTe Nanosheets Induced by Ligand Exchange, Chem. Mater., 2018, 30, 1710-1717.

23 S. Ithurria, G. Bousquet and B. Dubertret, Continuous Transition from 3D to $1 \mathrm{D}$ Confinement Observed during the Formation of CdSe Nanoplatelets, J. Am. Chem. Soc., 2011, 133, 3070-3077.

24 N. Tschirner, et al., Interfacial Alloying in CdSe/CdS Heteronanocrystals: A Raman Spectroscopy Analysis, Chem. Mater., 2012, 24, 311-318.

25 M. T. Frederick, V. A. Amin and E. A. Weiss, Optical Properties of Strongly Coupled Quantum Dot-Ligand Systems, J. Phys. Chem. Lett., 2013, 4, 634-640.

26 M. B. Teunis, S. Dolai and R. Sardar, Effects of SurfacePassivating Ligands and Ultrasmall CdSe Nanocrystal Size on the Delocalization of Exciton Confinement, Langmuir, 2014, 30, 7851-7858.

27 S. Jin, et al., Enhanced Rate of Radiative Decay in CdSe Quantum Dots upon Adsorption of an Exciton-Delocalizing Ligand, Nano Lett., 2014, 14, 5323-5328.

28 L. Lu, X.-L. Xu, W.-T. Liang and H.-F. Lu, Raman analysis of CdSe/CdS core-shell quantum dots with different CdS shell thickness, J. Phys.: Condens. Matter, 2007, 19, 406221.

29 V. M. Dzhagan, et al., Resonant Raman scattering study of CdSe nanocrystals passivated with CdS and ZnS, Nanotechnology, 2007, 18, 285701.

30 V. Dzhagan, et al., Morphology-induced phonon spectra of CdSe/CdS nanoplatelets: core/shell vs. core-crown, Nanoscale, 2016, 8, 17204-17212.

31 V. M. Dzhagan, Y. M. Azhniuk, A. G. Milekhin and D. R. T. Zahn, Vibrational spectroscopy of compound semiconductor nanocrystals, J. Phys. D: Appl. Phys., 2018, 51, 503001.

32 V. M. Dzhagan, et al., The influence of pyridine ligand onto the structure and phonon spectra of CdSe nanocrystals, J. Appl. Phys., 2011, 109, 084334.

33 Y. M. Azhniuk, et al., Resonant Raman studies of compositional and size dispersion of CdS 1- x Se x nanocrystals in a glass matrix, J. Phys.: Condens. Matter, 2004, 16, 90699082.

34 A. I. Lebedev, Lattice dynamics of quasi-two-dimensional CdSe nanoplatelets and their Raman and infrared spectra, 2017, pp. 1-7, DOI: 10.1103/PhysRevB.96.184306.

35 S. Singh, et al., Colloidal CdSe Nanoplatelets, A Model for Surface Chemistry/Optoelectronic Property Relations in Semiconductor Nanocrystals, J. Am. Chem. Soc., 2018, 140, 13292-13300.

36 B. Fritzinger, R. K. Capek, K. Lambert, J. C. Martins and Z. Hens, Utilizing Self-Exchange To Address the Binding of Carboxylic Acid Ligands to CdSe Quantum Dots, J. Am. Chem. Soc., 2010, 132, 10195-10201.

37 T. Djebaili, J. Richardi, S. Abel and M. Marchi, Atomistic simulations of the surface coverage of large gold nanocrystals, J. Phys. Chem. C, 2013, 117, 17791-17800.

38 H. Hinterwirth, et al., Quantifying thiol ligand density of self-assembled monolayers on gold nanoparticles by inductively coupled plasma-mass spectrometry, ACS Nano, 2013, 7, 1129-1136.

39 A. M. Smith, K. A. Johnston, S. E. Crawford, L. E. Marbella and J. E. Millstone, Ligand density quantification on colloidal inorganic nanoparticles, Analyst, 2017, 142, 11-29.

40 D. Chen, Y. Gao, Y. Chen, Y. Ren and X. Peng, Structure Identification of Two-Dimensional Colloidal Semiconductor Nanocrystals with Atomic Flat Basal Planes, Nano Lett., 2015, 15, 4477-4482. 